Draft Version July 18, 2011

Preprint typeset using $\mathrm{LATEX}_{\mathrm{E}}$ style emulateapj v. 11/26/03

\title{
A NEW INTERMEDIATE MASS PROTOSTAR IN THE CEPHEUS A HW2 REGION
}

\author{
Jesús Martín-Pintado, Izaskun Jiménez-Serra, Arturo Rodríguez-Franco ${ }^{1}$ \\ Dpto. de Astrofísica Molecular e Infrarroja, Instituto de Estructura de la Materia, Consejo Superior de Investigaciones Científicas \\ (CSIC), C/ Serrano 121, E-28006 Madrid, Spain; martin@damir.iem.csic.es, izaskun@damir.iem.csic.es, arturo@damir.iem.csic.es
}

Sergio Martín

Instituto de Radioastronomía Milimétrica, Avda. Divina Pastora, Local 20, E-18012 Granada, Spain; martin@iram.es

AND

Clemens Thum

Institut de Radio Astronomie Millimétrique, 300 Rue de la Piscine, F-38406 St. Martin d'Hères, France; thum@iram.fr Draft version July 18, 2011

\section{ABSTRACT}

We present the discovery of the first molecular hot core associated with an intermediate mass protostar in the Cep A HW2 region. The hot condensation was detected from single dish and interferometric observations of several high excitation rotational lines (from 100 to $880 \mathrm{~K}$ above the ground state) of $\mathrm{SO}_{2}$ in the ground vibrational state and of $\mathrm{HC}_{3} \mathrm{~N}$ in the vibrationally excited states $\mathrm{v}_{7}=1$ and $\mathrm{v}_{7}=2$. The kinetic temperature derived from both molecules is $\sim 160 \mathrm{~K}$. The high-angular resolution observations $\left(1.25^{\prime \prime} \times 0.99^{\prime \prime}\right)$ of the $\mathrm{SO}_{2} J=28_{7,21} \rightarrow 29_{6,24}$ line ( $488 \mathrm{~K}$ above the ground state) show that the hot gas is concentrated in a compact condensation with a size of $\sim 0.6^{\prime \prime}$ ( $\left.\sim 430 \mathrm{AU}\right)$, located $0.4^{\prime \prime}$ (300 AU) east from the radio-jet HW2. The total $\mathrm{SO}_{2}$ column density in the hot condensation is $\sim 10^{18} \mathrm{~cm}^{-2}$, with a $\mathrm{H}_{2}$ column density ranging from $\sim 10^{23}$ to $6 \times 10^{24} \mathrm{~cm}^{-2}$. The $\mathrm{H}_{2}$ density and the $\mathrm{SO}_{2}$ fractional abundance must be larger than $10^{7} \mathrm{~cm}^{-3}$ and $2 \times 10^{-7}$ respectively. The most likely alternatives for the nature of the hot and very dense condensation are discussed. From the large column densities of hot gas, the detection of the $\mathrm{HC}_{3} \mathrm{~N}$ vibrationally excited lines and the large $\mathrm{SO}_{2}$ abundance, we favor the interpretation of a hot core heated by an intermediate mass protostar of $10^{3} \mathrm{~L}_{\odot}$. This indicates that the Cep A HW2 region contains a cluster of very young stars.

Subject headings: ISM: individual (Cepheus A) — ISM: molecules — stars: formation

\section{INTRODUCTION}

While the general scenario of low mass star formation is reasonably well understood, the formation of massive stars is still poorly known (see e.g. McKee \& Tan 2003). Hot cores represent one of the earliest phases in massive star formation accretion (Garav \& Lizano 1999). They are compact condensations $(\leq 0.1 \mathrm{pc})$ with high densities $\left(\geq 10^{6} \mathrm{~cm}^{-3}\right)$, high kinetic temperatures $(\geq 100 \mathrm{~K})$ and a very rich chemistry. The abundances of sulfur-bearing molecules like $\mathrm{SO}_{2}$, are expected to be enhanced in hot cores by several orders of magnitude with respect to the quiescent gas (Charnlev 1997). So far, these hot cores have been mainly detected in objects whose luminosities are larger than $10^{4} \mathrm{~L}_{\odot}$ (Garav \& Lizano 1999).

Cepheus A (CepA), with a total IR luminosity of $2 \times 10^{4} \mathrm{~L}_{\odot}$, is a very active region of massive star formation. It contains sixteen ultracompact H II regions, molecular outflows and water vapor masers (e.g. Hughes \& Wouterloot 1984; Rodríguez, Moran. \& Ho 1980; Torrelles et al. 1996; Garav et al. 1996). The main powering source in the region is believed to be the thermal radio-jet, known as HW2, which is the brightest of the radio continuum sources in the region (Rodríguez et al. 1994; Garav et al. 1996). The surroundings of HW2 show a rather complex structure.

\footnotetext{
${ }^{1}$ Escuela Universitaria de Óptica, Departamento de Matemática Aplicada (Biomatemática), Universidad Complutense de Madrid, Avda. Arcos de Jalón s/n, E-28037 Madrid, Spain
}

The morphology of the HW2 radio-jet suggests the presence of a disk of neutral gas whose size is unknown. Torrelles et al. (1996) reported evidence for water masers in a rotating and collapsing disk around the radio-jet. However, the warm molecular gas (about $50 \mathrm{~K}$ ) around HW2 shows a ring like structure with complex kinematics which does not seem to be consistent with a simple rotating disk (Torrelles et al. 1999).

In addition to the expected neutral disk, within a few hundred $\mathrm{AU}$ of the radio-jet, there is clear evidence for very recent star formation. Two radio continuum sources detected by Curiel et al. (2002), one of them claimed to be the source of the expanding bubble found in $\mathrm{H}_{2} \mathrm{O}$ masers (Torrelles et al. 2001), are very likely protostars. Furthermore, the multiple outflow activity (Hayashi, Hasegawa, \& Kaifu 1988; Naravanan \& Walker 1996) seems to be powered by three different young stellar objects in the surroundings of HW2 (Codella et al. 2003). Two of these sources have not been yet identified.

In this letter, we report the detection of a hot $(160 \mathrm{~K})$, very dense $\left(\geq 10^{7} \mathrm{~cm}^{-3}\right)$ compact $\left(0.6^{\prime \prime} ; 430 \mathrm{AU}\right)$ condensation located $0.4^{\prime \prime}(300 \mathrm{AU})$ east from the radio-jet HW2. From the properties of the hot condensation and the large $\mathrm{H}_{2}$ column densities of hot gas, we conclude that this condensation is likely a hot core associated with an intermediate mass star with a luminosity of $10^{3} \mathrm{~L}_{\odot}$ which could be the driving source of one of the molecular outflows observed in the CepA region.

\section{OBSERVATIONS \& RESULTS}


The observations of the high excitation $\mathrm{SO}_{2}$ rotational lines and of the $\mathrm{HC}_{3} \mathrm{~N}$ vibrationally excited lines (hereafter $\left.\mathrm{HC}_{3} \mathrm{~N}^{*}\right)$ in Tab. 1 were made with the IRAM ${ }^{2} 30 \mathrm{~m}$ telescope at Pico Veleta (Spain). The lines were observed with the $1.3,2$ and $3 \mathrm{~mm}$ SIS receivers simultaneously. The half power beam width (HPBW) of the telescope ranged from $14^{\prime \prime}$ to $25^{\prime \prime}$. The receivers were tuned to single side band with image rejections larger than $10 \mathrm{~dB}$. The system temperatures of the $1.3,2$ and $3 \mathrm{~mm}$ receivers were, respectively, $\sim 450, \sim 310$ and $\sim 170 \mathrm{~K}$. As spectrometers, we used the VESPA autocorrelator, configured to provide velocity resolutions ranging from 0.037 to $3.3 \mathrm{~km} \mathrm{~s}^{-1}$. The line intensities were converted to main brightness temperatures using the beam efficiencies of $0.55,0.69$ and 0.77 for the $1.3,2$ and $3 \mathrm{~mm}$ lines respectively.

The $J=28_{7,21} \rightarrow 29_{6,24} \mathrm{SO}_{2}$ line $(489 \mathrm{~K}$ above the ground state) was also observed with high angular resolution using the IRAM Plateau de Bure Interferometer PdBI- (France) in the A and B configurations. The $3 \mathrm{~mm}$ receiver was tuned to double side band. The HPBW of the synthesized beam was $1.25^{\prime \prime} \times 0.99^{\prime \prime}, \mathrm{PA}=-25^{\circ}$. The correlator was configured to cover $145 \mathrm{MHz}\left(439 \mathrm{~km} \mathrm{~s}^{-1}\right)$ with a spectral resolution of $625 \mathrm{KHz}\left(1.89 \mathrm{~km} \mathrm{~s}^{-1}\right)$. CRL618 (1.73 Jy) and MWC349 (1.03 Jy) were used as flux density calibrators. The total observing time onsource was about 7 hours.

Fig. 1 shows the $\mathrm{SO}_{2}$ and $\mathrm{HC}_{3} \mathrm{~N}^{*}$ line profiles observed with the $30 \mathrm{~m}$ telescope and the PdBI toward CepA HW2, and Tab. 1 gives the line parameters obtained by fitting Gaussian profiles. Our $J=16_{2,14} \rightarrow$ $15_{3,13}$ and $J=3_{1.3} \rightarrow 2_{0.2} \mathrm{SO}_{2}$ lines are in agreement with those of Codella et al. (2003). The $J=3_{1,3} \rightarrow 2_{0,2}$ $\mathrm{SO}_{2}$ line will be not used in our analysis since it is easily excited and mainly arises from the extended molecular outflow. Like most of the low excitation lines of many molecules (Martín-Pintado, Bachiller, \& Fuente 1992; Bergin et al. 1997; Codella et al. 2003), all of the high excitation lines of $\mathrm{SO}_{2}$ and $\mathrm{HC}_{3} \mathrm{~N}^{*}$ peak around $10 \mathrm{~km} \mathrm{~s}^{-1}$. The $\mathrm{SO}_{2}$ lines also show a slightly asymmetric profile with a weak redshifted wing. This asymmetry is probably associated with the high velocity shocks observed in $\mathrm{SO}_{2}$ (Codella et al. 2003).

Fig. 2 shows the high angular resolution PdBI images of the continuum emission (thin solid contours and grey map) and of the $J=28_{7,21} \rightarrow 29_{6,24} \mathrm{SO}_{2}$ line intensity at $-10 \mathrm{~km} \mathrm{~s}^{-1}$ (thick solid contours). The continuum emission is unresolved in our beam with a peak continuum intensity of $110 \pm 15 \mathrm{mJy}$, in agreement with those previously measured by Mehringer, Zhou, \& Dickel (1997) and Gómez et al. (1999), and consistent with the continuum emission arising from the HW2 radio-jet. For all radial velocities, the $J=28_{7,21} \rightarrow 29_{6,24} \mathrm{SO}_{2}$ line emission is also spatially unresolved, indicating that the highly excited gas is very compact with a size of $\leq 0.6^{\prime \prime}$ (430 AU). The unresolved $\mathrm{SO}_{2}$ condensation contains most of the flux $(70 \%)$ measured with the $30 \mathrm{~m}$ telescope. As shown in Fig. 2, the $\mathrm{SO}_{2}$ emission is located $0.4^{\prime \prime}$ east from the radio-jet. This is consistent with the warm $\mathrm{NH}_{3}$ emission

\footnotetext{
2 The Institute for Radio Astronomy at Millimeter wavelengths (IRAM) is supported jointly by the German Max-Planck-Society, the French Centre National de Recherche Scientifique (CNRS) and the Spanish Instituto Geográfico Nacional.
}

observed in the $(3,3)$ line by Torrelles et al. (1999).

\section{PROPERTIES OF THE COMPACT MOLECULAR CONDENSATION}

Tab. 2 summarizes the properties of the compact molecular condensation. Fig. 3 shows the population diagrams (see e.g. Goldsmith \& Langer 1999) derived for $\mathrm{SO}_{2}$ and $\mathrm{HC}_{3} \mathrm{~N}^{*}$. The level populations of both molecules can be fitted by straight lines with similar excitation temperatures of $150-160 \mathrm{~K}$, indicating the presence of a hot condensation (hereafter $\mathrm{HC}$ ). We have constrained the size of the $\mathrm{HC}$ by using the upper limit to the size measured from the interferometric maps of $0.6^{\prime \prime}$, and the lower limit of $0.3^{\prime \prime}$ derived from the measured main beam brightness temperature of the $J=28_{7,21} \rightarrow 29_{6,24} \mathrm{SO}_{2}$ line and the excitation temperature of $160 \mathrm{~K}$. In the following discussion, we will consider a size of $0.6^{\prime \prime}$ (430 AU) for the HC. The derived excitation temperature from the $\mathrm{HC}_{3} \mathrm{~N}^{*}$ transitions is unlikely affected by optical depth effects since the observed lines are weaker than those expected from a source of $0.6^{\prime \prime}$ and optically thick emission with an excitation temperature of $160 \mathrm{~K}$.

Combining the column density derived from the PdBI data of the $J=28_{7,21} \rightarrow 29_{6,24} \mathrm{SO}_{2}$ line (see Table 1) with the estimated source size of $0.6^{\prime \prime}$ and the excitation temperature of $160 \mathrm{~K}$, we derive a total $\mathrm{SO}_{2}$ column density for the $\mathrm{HC}$ of $\sim 10^{18} \mathrm{~cm}^{-2}$. Although the $\mathrm{H}_{2}$ column density of the $\mathrm{HC}$ is unknown, it can be constrained between $10^{23}$ and $6 \times 10^{24} \mathrm{~cm}^{-2}$. The upper limit of $6 \times 10^{24} \mathrm{~cm}^{-2}$ is derived from our $3 \mathrm{~mm}$ continuum intensity of $30 \mathrm{mJy}$ measured at the location of the $\mathrm{HC}$, and by assuming that it arises from dust at a temperature of $160 \mathrm{~K}$. The lower limit is obtained by assuming the extreme case that $50 \%$ of the cosmic sulfur abundance is in $\mathrm{SO}_{2}$. Assuming spherical symmetry, the $\mathrm{H}_{2}$ densities in the $\mathrm{HC}$ range between $10^{7}$ and $6 \times 10^{8} \mathrm{~cm}^{-3}$. Hereafter (see Tab. 2), we will consider the intermediate case for a $\mathrm{H}_{2}$ column density of $10^{24} \mathrm{~cm}^{-2}$ (i.e. a $\mathrm{SO}_{2}$ abundance of $\left.10^{-6}\right)$. In this case, the $\mathrm{H}_{2}$ density and the mass of the $\mathrm{HC}$ will be $\sim 10^{8} \mathrm{~cm}^{-3}$ and $4 \times 10^{-2} \mathrm{M} \odot$ respectively. The kinetic temperature, $\left(\mathrm{T}_{\text {kin }}\right)$, of the $\mathrm{HC}$ is unknown, but it must be equal or larger than the derived excitation temperature of $160 \mathrm{~K}$. For the high density $\left(>10^{7} \mathrm{~cm}^{-3}\right)$ derived for the $\mathrm{HC}$, the $\mathrm{SO}_{2}$ transitions are thermalized and the excitation temperature should be a direct measurement of the kinetic temperature. Furthermore the dust and gas must be also closely coupled and the dust temperature should be equal to the gas kinetic temperature. Since the HC is optically thick in the mid-IR, we estimate a bolometric luminosity of $\sim 10^{3} \mathrm{~L}_{\odot}$ by assuming that it emits like a black body at $160 \mathrm{~K}$.

In summary, we have detected a warm $(160 \mathrm{~K})$, very dense $\left(>10^{7} \mathrm{~cm}^{-3}\right)$ and compact condensation (size $\sim 400 \mathrm{AU}$ ) located $300 \mathrm{AU}$ east from the radio-jet HW2. The HC has a total mass of $4 \times 10^{-2} \mathrm{M}_{\odot}$, a luminosity of $\sim 10^{3} \mathrm{~L}_{\odot}$ and a very large abundance $\left(\geq 2 \times 10^{-7}\right)$ of $\mathrm{SO}_{2}$.

\section{THE NATURE OF THE HOT CONDENSATION. A HOT CORE}

In view of the complexity of the region in the vicinity of HW2, we discuss the two most likely possibilities for the nature of the HC. The first one consists of a hot and dense spot in the expected circumstellar disk around the radio-jet. In the disk scenario, the large $\mathrm{H}_{2}$ column density $\left(10^{24} \mathrm{~cm}^{-2}\right)$ of hot gas $(160 \mathrm{~K})$ in the $\mathrm{HC}$ could be 
externally heated by the IR radiation from HW2 or by the shocks produced by the stellar wind of $\mathrm{HW} 2$. For the projected distance between HW2 and the HC, external heating by radiation would require the IR luminosity of HW 2 to be few $10^{5} \mathrm{~L} \odot$ (Fig. 7 of Kaufman, Hollenbach, \& Tielens 1998), which is at least one order of magnitude larger than that measured in the whole region of $2 \times 10^{4} \mathrm{~L} \odot$. Shock heating would require collisional excitation of the $\mathrm{v}_{7}=1$ and 2 vibrationally levels of $\mathrm{HC}_{3} \mathrm{~N}$ and the conversion of $10 \%$ of the total bolometric luminosity in the region into mechanical luminosity. Collisional excitation of the vibrational levels of $\mathrm{HC}_{3} \mathrm{~N}$ is possible since the $\mathrm{H}_{2}$ densities estimated for the $\mathrm{HC}$ are close to the critical densities of $2 \times 10^{8} \mathrm{~cm}^{-3}$ (Goldsmith et al. 1982). Shocks should then provide a mechanical luminosity of $10^{3} \mathrm{~L} \odot$ in a region of only $400 \mathrm{AU}$. This is very unlikely since the total mechanical luminosity in the large scale outflow powered by HW2 is only $30 \mathrm{~L} \odot$ (Naravanan \& Walker 1996), nearly two orders of magnitude smaller than the mechanical luminosity required to heat the HC.

The second possibility is that we are observing a hot core internally heated. This option will explain the presence of the vibrationally excited emission which in hot cores is usually produced by IR radiation (see e.g. de Vicente et al. 2000). The luminosity and the mass of the hot core in HW2 will be more than one order of magnitude smaller than those reported for hot cores associated with massive stars. Then, the $\mathrm{HC}$ will be the first hot core associated with an intermediate mass star. The luminosity of the hot core would require a main sequence B5 star. Large $\mathrm{SO}_{2}$ abundance $\left(\sim 10^{-7}\right)$ seems to be a general property of hot cores associated with massive protostars (Keane et al. 2001). Our lower limit to the $\mathrm{SO}_{2}$ abundance of $2 \times 10^{-7}$ suggests that intermediate mass hot cores have similar sulfur chemistry than those associated with massive protostars. The derived $\mathrm{HC}_{3} \mathrm{~N}$ abundance in the $\mathrm{HC}$ of $\sim 10^{-8}$, is also in agreement with the abundances measured in hot cores associated with massive stars (de Vicente et al. 2000). It has been proposed that the large abundance of sulfur-bearing molecules can be driven by fast gas phase reactions after the evaporation from grain mantles of key molecules like $\mathrm{H}_{2} \mathrm{~S}$ (Charnlev 1997), OCS (van der Tak et al. 2003; Martín et al. 2005) and SO (Jimenez-Serra et al. 2005) or atomic sulfur (Wakelam et al. 2004). From the available data on other sulfur-bearing molecules, $\mathrm{SO}_{2}$ seems to be the most abundant species in the $\mathrm{HC}$. We can set upper limits to the abundance of other sulfur- bearing molecules and of atomic sulfur by assuming that all their emissions observed with beams of $10-30^{\prime \prime}$, are optically thin and arise from the hot core. From the $\mathrm{H}_{2} \mathrm{~S}$, SO and [S I] $25.2 \mu \mathrm{m}$ observations of Codella et al. (2003), Martín-Pintado et al. (1992) and Wright et al. (1996) respectively, we derive column densities for the three species of $1-2 \times 10^{17} \mathrm{~cm}^{-2}$. Their corresponding abundances are $\leq 10^{-8}$, at least one order of magnitude smaller than the $\overline{\mathrm{S}} \mathrm{O}_{2}$ abundance. Viti et al. (2004) have performed model calculations to follow the sulfur chemistry evolution from the collapse phase to the hot core stage using new experimental data on desorption temperatures for mixed ices. The model predicts that an intermediate mass star of $5 \mathrm{M} \odot$ in the hot core phase will evaporate $\mathrm{H}_{2} \mathrm{~S}$ from the grain mantles, and after few $10^{5}$ years, will produce $\mathrm{SO}_{2}$ in gas phase with abundances similar to that observed in the intermediate mass hot core in HW2.

The discovery reported in this letter of an intermediate mass protostar, and the recent detection of two embedded young stellar objects (Curiel et al. 2002) within $500 \mathrm{AU}$ of HW2, support the scenario of the formation of a cluster of stars. The new protostar in the HW2 region is an excellent candidate for powering one of the three molecular outflows observed in the region (Codella et al. 2003). If the relationship found between the mechanical luminosity of the molecular outflows and the luminosity of their exciting sources (Shepherd \& Churchwell 1996) holds for the outflows in the CepA region, the new protostar will be luminous enough to power the east outflow (Havashi et al. 1988). However, high angular resolution observations of these outflows are needed to confirm if the new protostar is the driving source of one of the multiple outflows found in this region.

In summary, we have detected a hot and dense condensation in the vicinity of the radio-jet HW2 in the CepA region. From the properties of the hot condensation, we suggest that it is a hot core heated by an intermediate mass protostar. This protostar could be the driving source of one of the observed multiple molecular outflows. The detection of a new protostar in the vicinity of HW2 supports the idea of the formation of a cluster of massive and intermediate stars in the CepA region.

This work has been supported by the Spanish MEC under projects number AYA2002-10113-E, AYA200302785-E and ESP2004-00665.

\section{REFERENCES}

Bergin, E. A., Ungerechts, H., Goldsmith, P. F., Snell, R. L., Irvine, W. M., Schloerb, F. P. 1997, ApJ, 482, 267

Charnley, S. B. 1997, ApJ, 481, 396

Codella, C., Bachiller, R., Benedettini, M., \& Caselli, P. 2003, MNRAS, 341, 707

Curiel, S. et al. 2002, ApJ, 564, L35

de Vicente, P., Martín-Pintado, J., Neri, R., \& Colom, P. 2000, A\&A, 361, 1058

Garay, G., Ramírez, S., Rodríguez, L. F., Curiel, S., \& Torrelles, J. M. 1996, ApJ, 459, 193

Garay, G. \& Lizano, S. 1999, PASP, 111, 1049

Goldsmith, P. F., Snell, R. L., Deguchi, S., Krotkov, R., \& Linke, R. A. 1982, ApJ, 260, 147

Goldsmith, P. F. \& Langer, W. D. 1999, ApJ, 517, 209
Gómez, J. F., Sargent, A. I., Torrelles, J. M., Ho, P. T. P., Rodríguez, L. F., Cantó, J., \& Garay, G. 1999, ApJ, 514, 287 Hayashi, S. S., Hasegawa, T., \& Kaifu, N. 1988, ApJ, 332, 354 Hughes, V. A. \& Wouterloot, J. G. A. 1984, ApJ, 276, 204

Jimenez-Serra, I., Martín-Pintado, J. Rodríguez-Franco, A., \& Martín, S. 2005, submitted

Kaufman, M. J., Hollenbach, D. J., \& Tielens, A. G. G. M. 1998, ApJ, 497, 276

Keane, J. V., Tielens, A. G. G. M., Boogert, A. C. A., Schutte, W. A., \& Whittet, D. C. B. 2001, A\&A, 376, 254

Martín, S., Martín-Pintado, J., Mauersberger, R., Henkel, C., \& García-Burillo, S. 2005, ApJ, 620, 210

Martín-Pintado, J., Bachiller, R., \& Fuente, A. 1992, A\&A, 254, 315

McKee, C. F. \& Tan, J. C. 2003, ApJ, 585, 850 

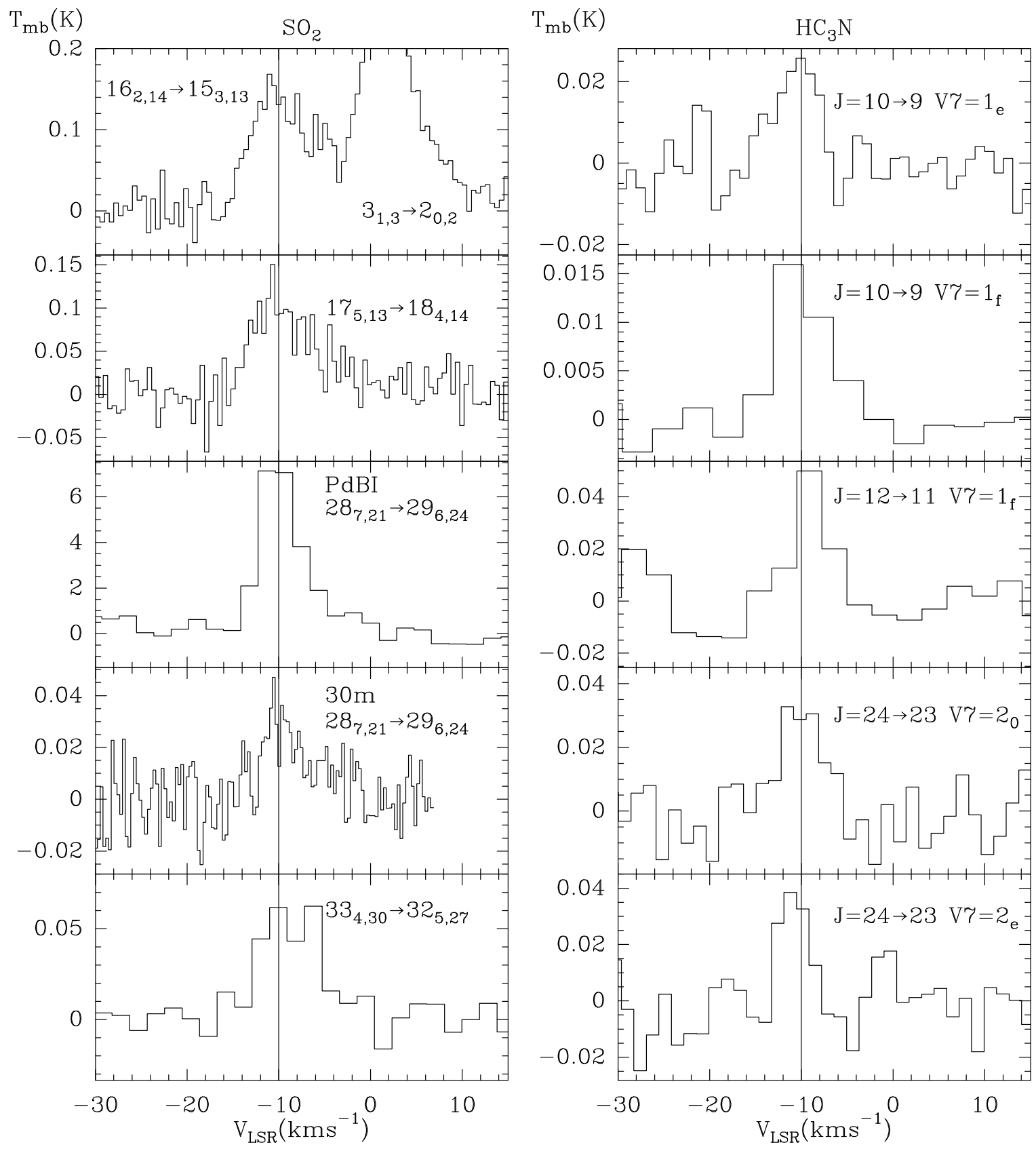

FIG. 1. - Sample of profiles of the $\mathrm{SO}_{2}$ and $\mathrm{HC}_{3} \mathrm{~N}$ vibrationally excited rotational transitions observed toward CepA HW2.

Mehringer, D. M., Zhou, S., \& Dickel, H. R. 1997, ApJ, 475, L57 Narayanan, G., \& Walker, C. K. 1996, ApJ, 466, 844

Rodríguez, L. F., Moran, J. M., \& Ho, P. T. P. 1980, ApJ, 240, L149

Rodríguez, L. F., Garay, G., Curiel, S., Ramírez, S., Torrelles, J. M., Gómez, Y., \& Velázquez, A. 1994, ApJ, 430, L65

Shepherd, D. S., \& Churchwell, E. 1996, ApJ, 472, 225

Torrelles, J. M., Gómez, J. F., Rodríguez, L. F., Curiel, S., Ho, P. T. P., \& Garay, G. 1996, ApJ, 457, L107

Torrelles, J. M., Gómez, J. F., Garay, G., Rodríguez, L. F., Miranda, L. F., Curiel, S., \& Ho, P. T. P. 1999, MNRAS, 307, 58
Torrelles, J. M., et al. 2001, Nature, 411, 277

van der Tak, F. F. S., Boonman, A. M. S., Braakman, R., \& van Dishoeck, E. F. 2003,A\&A, 412, 133

Viti, S., Collings, M. P., Dever, J. W., McCoustra, M. R. S., \& Williams, D. A. 2004, MNRAS, 354, 1141

Wakelam, V., Caselli, P., Ceccarelli, C., Herbst, E., \& Castets, A. 2004, A\&A, 422, 159

Wright, C. M., Drapatz, S., Timmermann, R., van der Werf, P. P., Katterloher, R., \& de Graauw, T. 1996, A\&A, 315, L301 


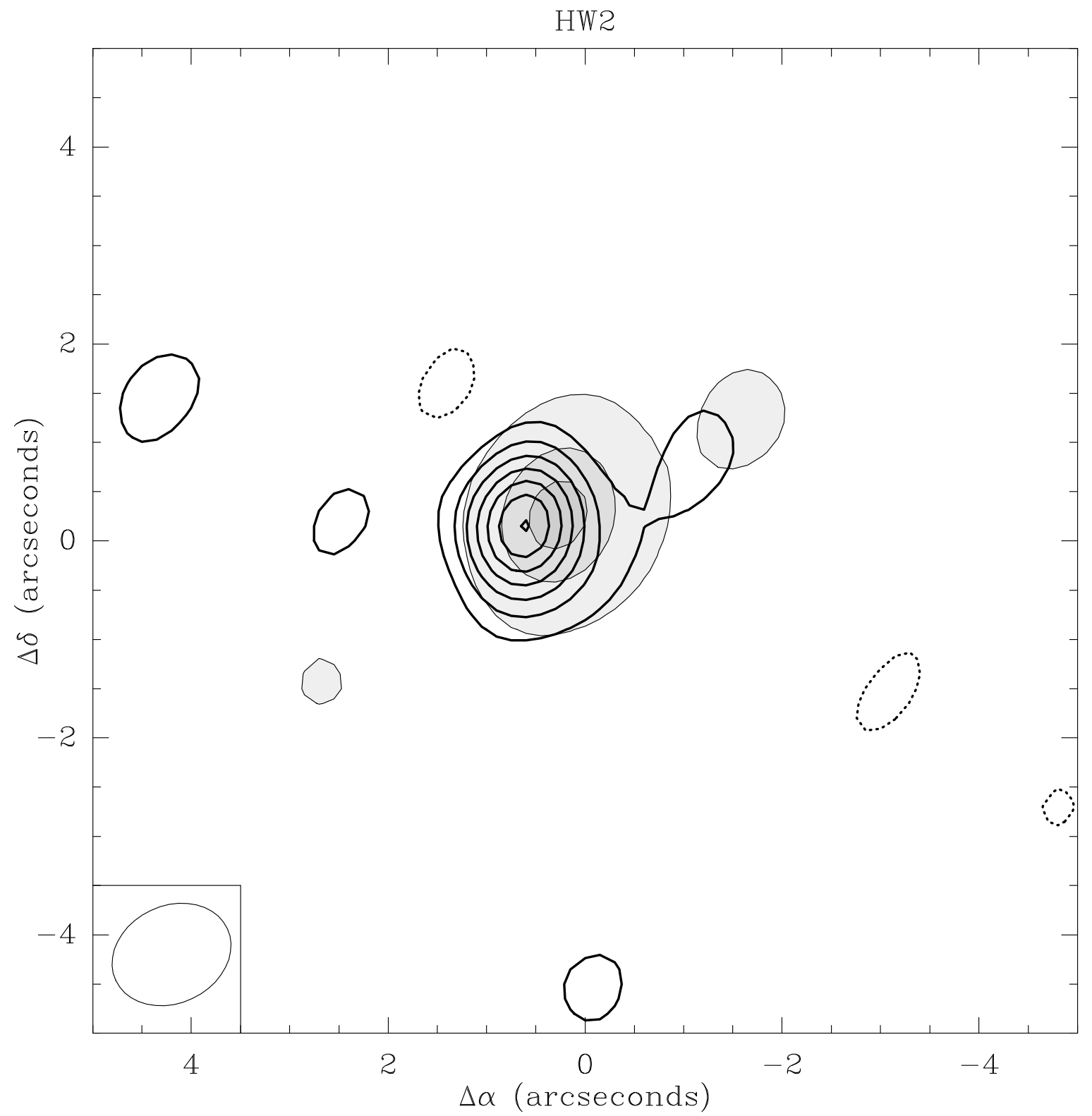

FIG. 2.- Images of the $J=28_{7,21} \rightarrow 296,24 \mathrm{SO}_{2}$ line intensity at $-10 \mathrm{~km} \mathrm{~s}^{-1}$ (thick solid contours) superimposed on the radio continuum emission at $3 \mathrm{~mm}$ (thin solid contours and grey map). The lowest level and the step for the continuum map are $0.01 \mathrm{Jy} / \mathrm{beam}$ and $0.04 \mathrm{Jy} /$ beam respectively. The contour levels for the $\mathrm{SO}_{2}$ map are $-0.01,0.01,0.02,0.03,0.04,0.05,0.06$ and $0.07 \mathrm{Jy} / \mathrm{beam} \mathrm{km} \mathrm{s}^{-1}$. Beam size is shown in the lower left corner. 


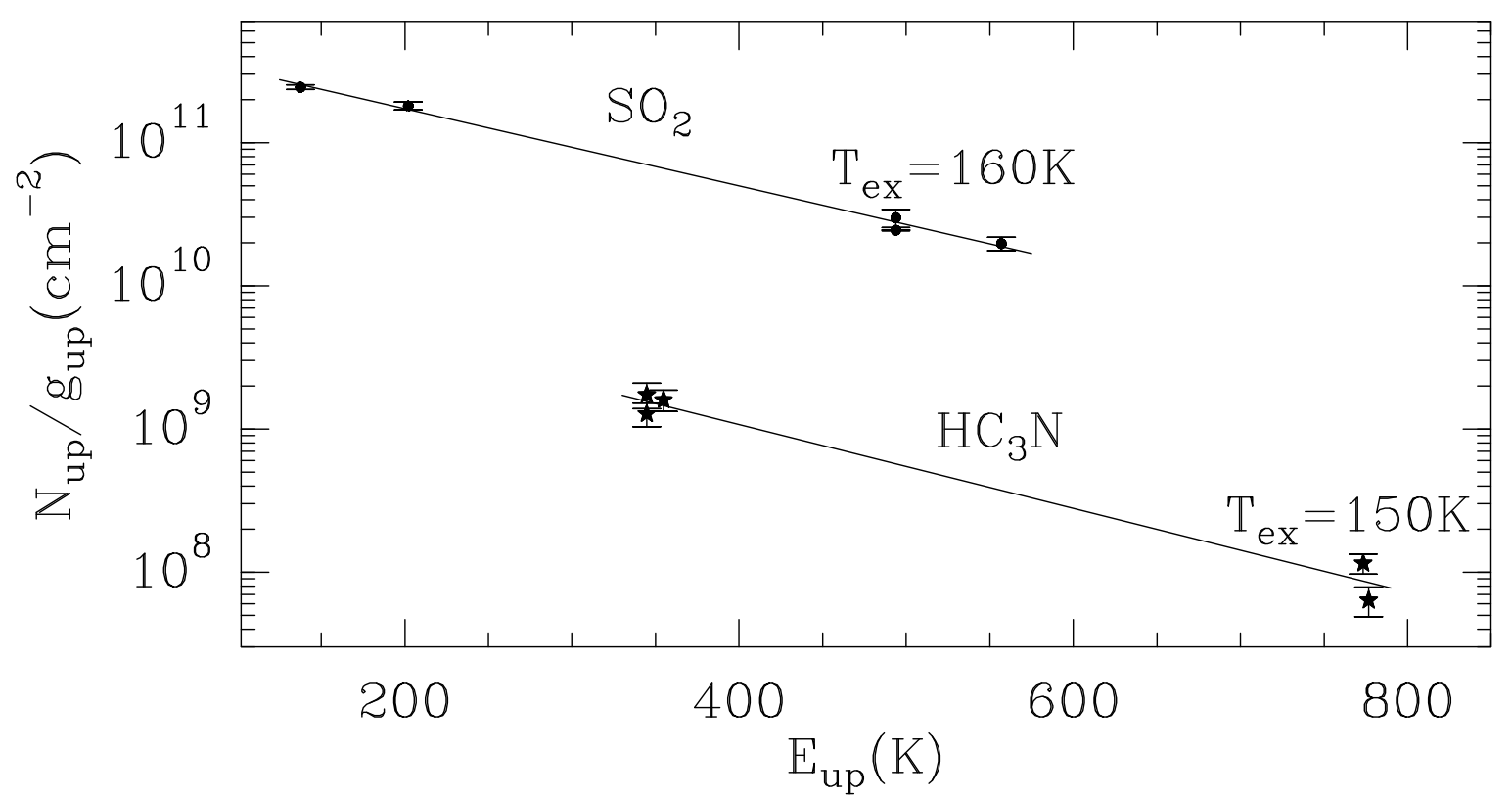

FIG. 3.- Population diagrams for all the $\mathrm{SO}_{2}$ and $\mathrm{HC}_{3} \mathrm{~N}^{*}$ rotational transitions observed toward CepA HW2. The column densities are averaged values in a beam of $24^{\prime \prime}$ for $\mathrm{SO}_{2}$ and $27^{\prime \prime}$ for $\mathrm{HC}_{3} \mathrm{~N}^{*}$. 


\begin{tabular}{lcccc}
\hline \hline Line & $\begin{array}{c}\mathrm{V}_{L S R^{\mathrm{a}}} \\
\left(\mathrm{km} \mathrm{s}^{-1}\right)\end{array}$ & $\begin{array}{c}\Delta v^{\mathrm{a}} \\
\left(\mathrm{km} \mathrm{s}^{-1}\right)\end{array}$ & $\begin{array}{c}\mathrm{T}_{m b}{ }^{\mathrm{a}} \\
(\mathrm{K})\end{array}$ & $\begin{array}{c}N_{u p} \\
\left(\times 10^{11} \mathrm{~cm}^{-2}\right)\end{array}$ \\
\hline & & $\mathrm{SO}_{2}$ & \\
$16_{2,14} \rightarrow 15_{3,13}$ & $-9.7(1)$ & $7.9(4)$ & $0.144(3)$ & 80.4 \\
$175,13 \rightarrow 184,14$ & $-9.6(3)$ & $7.8(6)$ & $0.100(3)$ & 70.3 \\
$28,21 \rightarrow 296,24$ & $-10.0(1)$ & $5.2(3)$ & $8.8(1)$ & $3628^{\mathrm{b}}$ \\
$28,21 \rightarrow 296,24$ & $-9.4(5)$ & $6(1)$ & $0.030(4)$ & 15.4 \\
$33_{4,30 \rightarrow 322_{5,27}}$ & $-8.8(4)$ & $7.8(9)$ & $0.060(4)$ & 30.0 \\
$10 \rightarrow 9 v_{7}=1 e$ & $-10.5(5)$ & $4.8(9)$ & $0.026(6)$ & 0.36 \\
$10 \rightarrow 9 v_{7}=1 f$ & $-10.3(5)$ & $5(2)$ & $0.017(4)$ & 0.27 \\
$12 \rightarrow 11 v_{7}=1 f$ & $-8.9(5)$ & $4.3(8)$ & $0.050(3)$ & 0.57 \\
$24 \rightarrow 23 v_{7}=20$ & $-10.2(5)$ & $7(1)$ & $0.04(1)$ & 0.33 \\
$24 \rightarrow 23 v_{7}=2 e$ & $-10.8(4)$ & $3.4(8)$ & $0.04(1)$ & 0.18 \\
& & & &
\end{tabular}

${ }^{\text {a The }} 1 \sigma$ errors from the gaussfits are shown in parenthesis.

${ }^{\mathrm{b}} \mathrm{SO}_{2}$ column density derived for a beam of $1.25^{\prime \prime} \times 0.99^{\prime \prime}$.

\begin{tabular}{|c|c|c|c|c|c|c|c|c|}
\hline \multirow[t]{2}{*}{ Source } & \multirow{2}{*}{$\begin{array}{l}\text { Location }^{\mathrm{a}} \\
\left({ }^{\prime \prime}\right)\end{array}$} & \multirow{2}{*}{$\begin{array}{c}\text { Size } \\
\left({ }^{\prime \prime}\right)\end{array}$} & \multirow{2}{*}{$\begin{array}{c}\mathrm{T}_{k i n} \\
(\mathrm{~K})\end{array}$} & \multirow{2}{*}{$\begin{array}{c}\mathrm{n}_{\mathrm{H}_{2}} \\
\left(\mathrm{~cm}^{-3}\right)\end{array}$} & \multicolumn{2}{|c|}{ Column densities ${ }^{\mathrm{b}}$} & \multirow{3}{*}{$\begin{array}{c}\begin{array}{c}\text { Luminosity } \\
\left(\mathrm{L}_{\odot}\right)\end{array} \\
1 \times 10^{3}\end{array}$} & \multirow{2}{*}{$\begin{array}{l}\text { Mass } \\
\left(\mathrm{M}_{\odot}\right)\end{array}$} \\
\hline & & & & & $\mathrm{SO}_{2} \quad \mathrm{HC}_{3} \mathrm{~N}$ & $\mathrm{H}_{2}{ }^{\mathrm{c}}$ & & \\
\hline $\mathrm{HC}$ & $0.32(0.03) \times 0.15(0.03)$ & $0.3-0.6$ & $\overline{160(10)}$ & $1.5 \times 10^{8}$ & $3 \times 10^{16}$ & $1 \times 10^{24}$ & & \\
\hline
\end{tabular}

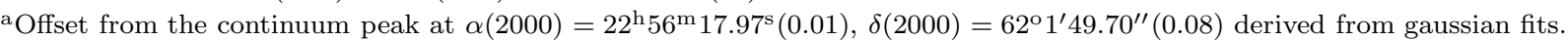

${ }^{\mathrm{b}}$ For a source size of $0.6^{\prime \prime}$ (430 UA) and a distance to the source of $725 \mathrm{pc}$. Column densities are in units of $\mathrm{cm}^{-2}$.

${ }^{\mathrm{c}}$ See text for details. 